\title{
Design and Implementation of A Bicycle Parking System Based on Internet-of-things
}

\author{
Yujia Huang \\ School of Electronics and Information \\ Engineering, Sichuan University \\ Chengdu, China, 15680797321 \\ yjhuang327@126.com
}

\author{
Zhongliang Yang \\ School of Electronics and Information \\ Engineering, Sichuan University \\ Chengdu, China
}

\author{
Shuhua Xiong \\ School of Electronics and Information \\ Engineering, Sichuan University \\ Chengdu, China
}

\begin{abstract}
How to manage increasing bicycles in a university campus is a challenging problem. To solve the problem, we design and implement an intelligent bicycle parking system based on the Internet of things. The system architecture is described, and the system modules are introduced. Moreover, we present the methodology used to implement the system. Finally, we give a deployment instance of the system, and discuss its possible applications. It is believed that the intelligent bicycle parking system has a brand application prospect.
\end{abstract}

Key words: Bycycle Parking system; Internet of Things; Radio Frequency Identification

\section{INTRODUCTION}

IoT has attracted significant concern, since it was defined by MIT Auto ID team in 1999 [1]. The full name of IoT is "the Internet of Things", which means it's a kind of specific network that has capacity to connect with any physic things. Indeed, its' core and fundament are still based on the communication of the Internet. However, it's the brand new extension of virtual Internet in physical world. Moreover, combining Radio-Frequency-Identification (RFID) technique, any physical thing connected to the IoT can communicate information with other things. Also, through connecting with computer networks, IoT can achieve automatic identification and information sharing of goods or commodities, which benefits a lot to management of mega markets. Thus, we can't ignore the giant potential of IoT on physical managements.

In recent years, with the extension of the area of university campus in China, many students and teachers prefer to ride bicycles. With the increasing of bicycle number, management of bicycles becomes a difficult problem. That is why more and more bicycle thefts are emerging in campuses. Fortunately, the popularization of IoT provides a good opportunity to solve this problem. In fact, the application of IoT has a profound signification to the digitization and standardization of bicycles management on campuses [2,3]. In this paper, we present a novel bicycle parking system based on IoT, which can record and process the all-around information of parking sites as well as the information of bicycles parking in the carport. This system will make the management of bicycles easier, and be useful to reduce the bicycle thefts.

The rest of this paper is organized as follows. Section 2 describes the architecture of the parking system based on IoT.
Section 3 presents the implementation and deployment of the whole system. Section 4 gives conclusions.

\section{The Architecture of The Bicycle PARking SYSTEM BASED ON IOT}

\section{A. System Architecture}

As Fig. 1 shows, the bicycle parking system consists of 3 subsystems, i.e., bicycle carport subsystem, controlling and information subsystem and alarming subsystem. The bicycle carports subsystem plays a leading role as the main serves, such as parking and inquiring. There are two main components in this subsystem, namely, a series of electrified locksets and corresponding inquiry machines. Each electrified lockset or inquiry machine is connected through an Ethernet interface [4] to a TCP/IP switcher that is connected with the controlling servers in the Control Center. The main function is to detect working states and record all signals that come from the electronic locksets. Besides, the inquiring requests received by the controlling and information subsystem can also come from the inquiry machines, which are expected to work the whole day. The controlling servers are linked with the information system where the entire bicycle uses' information stores. The alarm system connected with the controlling servers can send SMS alarms to Users automatically [5] or notify staffs in emergent situations. In addition, a website will be set up, and all parking information, e.g., the number of empty parking sites and the parking address of particularly users can be uploaded. Thereby, users can get information about their bicycles and the working situation of carports through the internet.

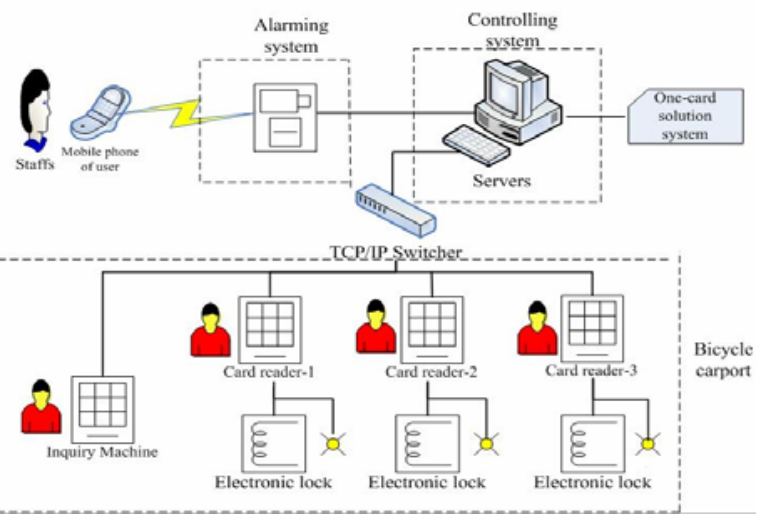

Fig.1 The architecture of the system 


\section{B. System Modules}

The bicycle parking system is a cooperative work of five different parts, i.e., identity machine, control center, electrified locksets, alarm machine and query machine, as Fig. 2 shows.

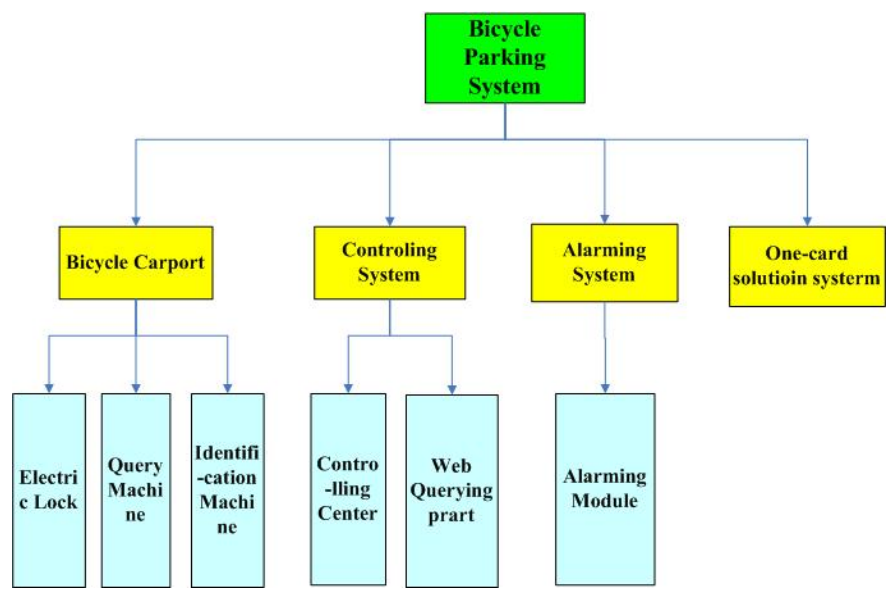

Fig. 2 The function modules of the system

The identity machines used to confirm the identity card of users, and record their information. Its core is a RFID card reader where users can swipe their cards to enter the bicycle parking system. This machine can accurately recognize different kinds of card, and only allow the authorized access.

The electrified locksets part is made up of a series of electrified locksets connected with RFID card readers. The card readers have two functions. One is to judge whether the user want to park or fetch their bicycle when they use this system. The other is to distinguish if the cardis unauthorized. After users swipe their cards on the card reader, the machine will send the signal to the electrified locksets to make them close or pen. Besides, the card readers also have contact with the control center through the Ethernet. Thus, the particular electrified lockset can be under the control of the control center, which makes it possible for administers to manage the whole system.

The query machine is designed to make it easier for users to find out where their bicycles parking or where has an available place to park, which can save the time spend on finding them. What Users have to do is just swipe their cards on the machine. If the machine judges that the user want to park, it will shows all the empty parking sites in the carports; if the machine judges the user comes to get the bicycle, it will display where the bicycle is. It is useful in the case of forgetting the place where the bicycle is parked.

This alarm machine is designed to alarm the managers and users when some special circumstances happen. It has two different methods to make the manager and users know what is happening. When some emergencies happen, such as power-off, illegal opening, malicious damage, three consecutive input wrong passwords or some other problems, the machine will send SMS to the user and the stuffs at the first time. At the same time, it will send out some other kinds of alarm signals like the alarm indicator on cabinet gets bright and the sirens scream, so the administers in control center will also know about it, which is useful for people to find out the problems and solve the breakdowns as soon as possible.

The main function of the Control Center is to manage and recode the users' information. This part is the key of the whole system. We will introduce it in detail in the following articles. The control center is built up by several servers, which have a connection with other parts and record all the information of working status from other modules.

\section{IMPLEMENTATION OF THE BICYVLE PARKING SYSTEM}

\section{A. Hardware Implementation}

To realize our design, we began our research with special improvement of a well-designed and premium product, SC102 entrance guard system made by Zksoftware Company. SC102 can provide three different modes of operation, i.e, the most convenient use induction card operation, the most accurate induction card +5 bit code and the most simple with only 1 to 5 bit cipher operation.

To adapt this product and realize more specific functions, we installed the Software Development Kit and resolved the hard devices of system. Then, as we designed, we adapted the original devices into two kinds of machines: inquiring machine and electrified locksets. Specially, in the adaption of electrified locksets, we add a loop circuit to the key part of lock, so that, if the lock is forcedly opened, the loop circuit will be cut-off, which will trigger the alarm system immediately. Another innovation in the inquiring machine's adaption is the equipment of guide lamps. When a user is inquiring the address of a bicycle, the number of the specific parking site will not only be printed on the screen, but also be indicated by the guide lamps. Thus, the user can find their bicycles or empty parking site as soon as possible.

To set up an intelligent network, we establish several servers as controlling center, which connect with all the TCP/IP switchers. In the Controlling Center, the servers run a specific SDK program adapted by our programmers to record and control all the electrified locksets and inquiring machines in carports. What's more, the servers also link to a SMS \& Sound alarm system, which can notify the emergency situation to both staffs and users while their bicycles are in dangerous of being stolen. In addition, to cooperate with one-card solution on campuses, the servers also connect to their particular network. Therefore, the whole system is divided into several parts, and each part performs different functions.

Another problem to be solved is electricity supply. To minimize the cost of carports, most of intelligent carports are connected with grids. Besides, in some particular area, due to the low power cost of the system, the carport can also rely on the solar battery. To design a perfect product, the carport also equipped with backup batteries to avoid unforeseen power cuts. The backup batteries are also under the monitoring of controlling center. 


\section{B. Software Implementation}

To utilize and improve the equivalent functions provided by original devices, the only adaption on hardware isn't enough. So, we also adapt and improve the software both on devices and servers. In the devices, we got the source code of original products, and then, according to communication protocol and TFT SDK manual offered by Zksoftware company, we figured out the meaning and functions of particular code. After that, we adapted the source code and upload our new code to the devices to achieve our planned functions. These functions help us complete the totally workflow, as Fig. 3 shows:

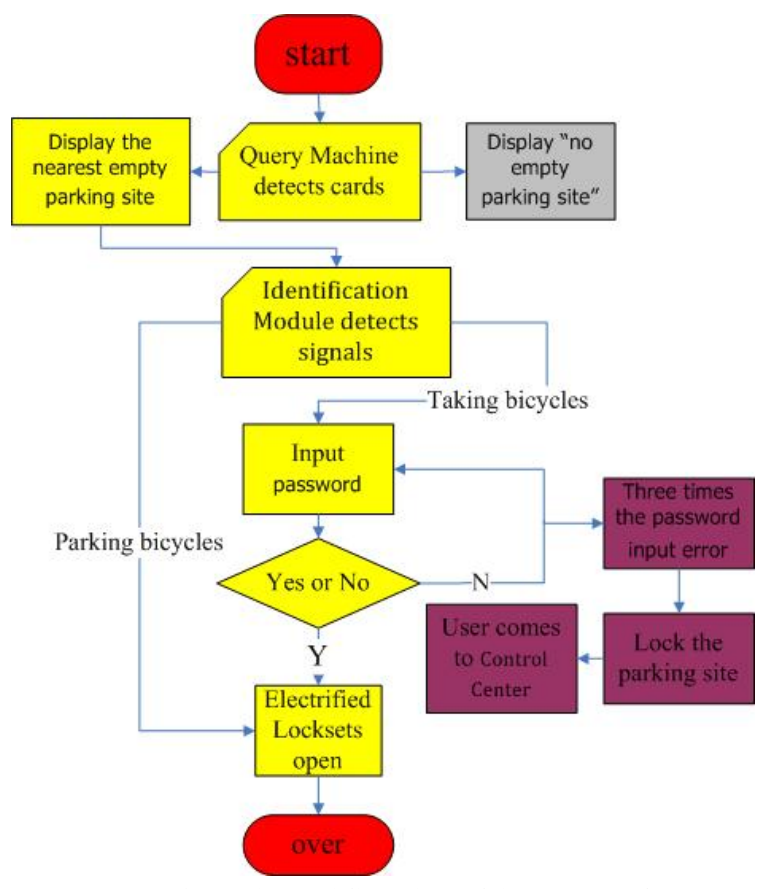

Fig. 3 The workflow chart of the system software

The system begins with the detection of cards, and then our software will get the information of cards. So, the system will offer the necessary information to users about the particular address of their bicycles or the places of empty parking sites by inquiring machines. The software can distinguish the specific purpose of users by reading the history records of users from data banks in controlling center. Afterwards, at the electrified locksets, the software will guide the user to park the bicycle or take their bicycle. If the user is going to park their bicycles, the lockset will move to parking process. If the software considers that the user is going to take their bicycle, the lockset will require the user to input a password. Once the password is confined, the lock will open and immediately. But, if the password is incorrect, the lockset will continue locking, and send the specific users' information to controlling center and trigger the SMS alarm system.

In servers, we use another program----ZKTime 5.0 Version 4.8.5, which was previously deigned to manage the entrance door system and record all the history of opening doors. Now, we improve the function of the original programs. The new program can not only connect and change information with official one-card solution system, but also record and detect the states of all electrified locksets in 24 hours. What is more, the servers can also answer the information quest and inquiry from inquiring machines. In other words, the controlling center is a document library, which stored all the information and history of the processes. Owning the prior authority, controlling center can command any locksets at any time in remote manner. Thus, staffs can solve any problems happened in any carports. Further, to maximize the capacity of servers, we set several special servers that host a specific web site, where users can look up the state and address of their bicycle. Also, the website can show the number of empty parking sites at any carport. As we believe, this website will help peoples a lot.

C. The State Machine of the system

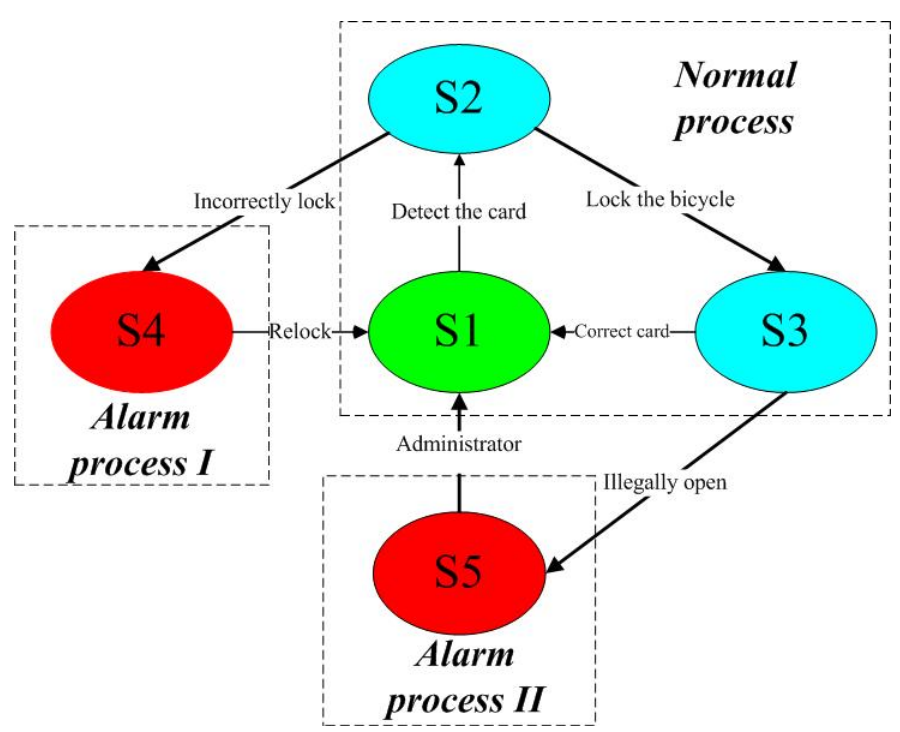

Fig 4.The states of the system

The system has totally 5 different kinds of states:

S1: Original State: the lockset is standby and waiting for providing services.

S2: Locking State: the lock is opening for a few seconds and it is time to put the bicycle into parking site correctly.

S3: The user locks the bicycle manually.

S4: The bicycle is not locked correctly or the lock is still opened.

S5: Illegally or forcibly open the lock.

The complete working states seem very complicate. In fact, the whole working states can be categorized in to three different kinds of processes.

A) The first one is obviously the Normal Process. In this process, there are three different working states. First, the lockset is standby and the lock is opened. The guide lamp also lights to show that it's an available parking site. Then S1 $\rightarrow$ S2 happens, when the card reader in lockset detects the signals from card. In S2, the lock 
will light a green lamp to tell the user it's time to put their bicycle in to parking site and the guide words will also be printed on the screen of lockset. After locking the bicycle rightly, S2 $\rightarrow$ S3 is going to happen. The S3 is the storing state of the bicycle. Now, the user's bicycle is locked safely in the parking site and also under the monitoring of alarm system. Finally, Then $\mathrm{S} 3 \rightarrow \mathrm{S} 1$, when the card reader confirms the correct information of the user. The lock will be open again and the parking site will be also available again in the record of controlling center.

B) Alarm Process I is a specific state, which includes two specific situations. One is the most common situation $\mathrm{S} 2 \rightarrow \mathrm{S} 4$. If the lockset detects that the lock stays at the S2 for a long time, then lockset will switch to the S4 and lights a red alarm lamp to notify staffs and users that the bicycle may be locked in an incorrect way. The other situation is simple, namely, the lock may still be opened. To solve the two situations, the administrators or users just need to press the "Cancel” bottom on the lockset to skip to the S1 and then relock the bicycles.

C) Alarm Process II is also a particular state and represents a quite emergency situation. When the lockset switches to S5 from S3, it means the lock is forcibly or illegally opened. In this state, the lockset will not only trigger the alarm bells and lamps but also send signals to controlling center to wake up SMS alarm system. Thus the users will receive the alarm message. In some particular conditions, the SMS alarm system can also notice polices directly. In addition, only administrators have the authority to switch the lockset back to S1. Thus, thieves are impossible to shut down the alarm system.

\section{Deploy and Application of the System}

The system is designed to deploy on campuses, especially the mega campuses. In the standard module, there will be several intelligent carports located dispersedly all over the place. Of course, we can set more carports near the specific places such as teaching buildings, dining halls and dormitories. What's more, it's considerably important to follow the willing of the residents in campuses. Therefore, we will have a deeply research in individual campus before choosing the addresses of the carports. Moreover, after the establishment of individual intelligent parking systems in different campuses, we plan to unite all the parking systems nearby, so that people can park their bicycles in different campuses, which will be supreme convenient. It is worth to note that the proposed system can not only apply on the campuses, but also contribute a lot at some specific places like commodities, mega playground such like Disneyland and also some small towns.

Therefore, the application of intelligent parking system contains enormous potential. In the future, based on the great advantages of our system, we can further develop a brand new bicycle rental service. To achieve this goal, we do not need to adapt our hardware a lot, and just need to implement the management of rental bicycles and accounting of rental fee in software. Therefore, the parking system is a developing but potential valuable product.

Although many companies have tried a lot on the emerging market of bicycle rental service, few projects succeeded finally. To avoid the similar tragedy, we plan to start our service at the campuses first, where people are well educated. Another great advantage of our application is that users' information is all based on one-card solution data banks, which is similar like credit card system in banks. Each individual has a reputation and credit record. The loss of damaged bicycles will be compensated by specific people. The more bad credit one gets, the less services he can gain.

In sum, the proposed intelligent parking system has a broad application prospect.

\section{CONCLUSIONS}

With the increasing of the bicycle number in universities, the effective management of bicycles becomes a significant and challenging problem. To solve the problem, using the IoT technology, we design and implement an intelligent bicycle parking system. This paper introduces the architecture and modules of the system, and presents the methodology of system implementation. This system will be deployed on our campus. However, it is worth noting that this system can also be applied in other open places, such as Disneyland and also some small towns. In the future, we will further improve this system and try to apply it in other areas.

\section{ACKNOWLEDGEMENT}

The work is supported by the innovation practice program for the undergraduate of China (No:201210610166).

\section{REFERENCES}

[1] DEAN, J., AND GHEMAWAT, S. Mapreduce: simplified data processing on large clusters. In OSDI'04: Proceedings of the 6th conference on Symposium on Opearting Systems Design \& Implementation (Berkeley, CA, USA, 2004), USENIX Association, pp. 10-10.

[2] XING XU, XINGNIN SU, LU YAO. The Research of One Card Solution. Laboratory Research \& Exploration, Beijing, China, 2004,No.3

[3] GARFINKEL, S. An Evaluation of Amazon's Grid Computing Services: EC2, S3 and SQS . Tech. Rep. TR-08-07, Harvard University, August 2007.

[4] GHEMAWAT, S., GOBIOFF, H., AND LEUNG, S.-T. The google file system. In SOSP '03: Proceedings of the nineteenth ACM symposium on Operating systems principles (New York, NY, USA, 2003), ACM, pp. 29-43.

[5] ZHIHONG WANG, ZENGTAO XUE, ZHIJUN CHEN, SHENHUI DU. The design of intelligent lock. Modern Electronic Technology,Beijing, China, 2007 No.9.

[6] GRAY, J., AND PATTERSON, D. A conversation with Jim Gray. ACM Queue 1, 4 (2003), 8-17.

[7] HAMILTON, J. Internet-Scale Service Efficiency. In LargeScale Distributed Systems and Middleware (LADIS) Workshop,September 2008. 